\title{
Spatial and Temporal Scaling of Thermal Infrared Remote Sensing Data
}

\author{
DALE A. QUATTROCHI
}

National Aeronautics and Space Administration, George C. Marshall Space Flight Center, Space Sciences Laboratory, Earth System Science Division, ES44, Marshall Space Flight Center, AL 35812, USA

NARENDRA S. GOEL

Department of Computer Science, Wayne State University, Detroit, MI 48202, USA

\section{ABSTRACT}

Although remote sensing has a central role to play in the acquisition of synoptic data obtained at multiple spatial and temporal scales to facilitate our understanding of local and regional processes as they influence the global climate, the use of thermal infrared (TIR) remote sensing data in this capacity has received only minimal attention. This results from some fundamental challenges that are associated with employing TIR data collected at different space and time scales, either with the same or different sensing systems, and also from other problems that arise in applying a multiple scaled approach to the measurement of surface temperatures. In this paper, we describe some of the more important problems associated with using TIR remote sensing data obtained at different spatial and temporal scales, examine why these problems appear as impediments to using multiple scaled TIR data, and provide some suggestions for future research activities that may address these problems. We elucidate the fundamental concept of scale as it relates to remote sensing and explore how space and time relationships affect TIR data from a problem-dependency perspective. We also describe how linearity and non-linearity observation versus parameter relationships affect the quantitative analysis of TIR data. Some insight is given on how the atmosphere between target and sensor influences the accurate measurement of surface temperatures and how these effects will be compounded in analyzing multiple scaled TIR data. Last, we describe some of the challenges in modeling TIR data obtained at different space and time scales and discuss how multiple scaled TIR data can be used to provide new and important information for measuring and modeling land-atmosphere energy balance processes. 


\section{INTRODUCTION}

The determination of surface temperature is a critical component in calculating the energy balance constituents of land-atmosphere interactions. This is particularly important in measuring and modeling boundary layer energy balance processes such as soil moisture, evapotranspiration, and fluxes of sensible and latent heat, and their partitioning across the surface of the Earth. Remote sensing provides a means for the synoptic measurement of these land-atmosphere energy fluxes and also offers the potential for understanding how different temperature fields are scaled between different spatial and temporal resolutions (Goetz et al., 1993 , 1994). Thus, using remote sensing to both observe and measure changes in fluxes of sensible and latent heat as a response to changes in spatial or temporal scale, will result in the development of better and more accurate models of these fluxes as they affect local, regional, and even global predictions of climatic variations. Remote sensing has a central role to play in the acquisition of synoptic data collected at multiple spatial and temporal scales that will facilitate our understanding of local scale processes (e.g., catchment watershed basin characteristics) as they influence regional scale processes (e.g., stream and river basin hydrodynamics). In fact, analysis of multiple spatial and temporal scaled remote sensing data is critical to understanding the coupling between the terrestrial landscape and the atmosphere (Hall et al., 1988).

It has been suggested that the use of multiple scale thermal infrared (TIR) remote sensing data could provide information on important cultural, geological, and agricultural variables (Lynn, 1986; Schott, 1989). To date, however, the use of multiple scaled TIR data to analyze Earth-surface characteristics and processes has not been fully exploited. This results from some fundamental challenges that are associated with using TIR data obtained at different spatial and temporal scales, both from the same and different sensors, as well as some basic problems that arise in applying a multiple scaling approach to the measurement of surface temperatures. In this paper, we wish to describe some of the more important problems associated with using TIR remote sensing data obtained at different spatial and temporal scales, examine why these problems appear as impediments to using multiple scaled TIR data, and provide some direction for future research that may resolve these problems. We will present these points from a general viewpoint and will not delve into some of the more complicated issues concerned with TIR data analysis, such as emissivity and the problems associated with calculating emissivity from TIR data acquired at different space and time scales. We approach our discussion by first describing the fundamental concept of scale as it relates to remote sensing. Such a discussion is necessary to define what is meant by scale, from both a spatial and temporal sense, as a foundation for understanding what happens when data from either the same, or different, sensors are combined together for multiple scaled analyses. We then discuss how space and time relationships affect TIR data from a problem-dependency perspective and describe how linearity and non-linearity observation versus parameter relationships affect the quantitative analysis of TIR data. We also present some insight 
on how the atmosphere between target and sensor influences the accurate measurement of surface temperatures and how these effects will be compounded in analyzing multiple scaled TIR data. Last, we describe some of the challenges in modeling TIR data obtained at different space and time scales and offer some insight into how multiple scaled TIR data can be used to provide new and important information for measuring and modeling land-atmosphere energy balance processes.

\section{THE FUNDAMENTAL ELEMENTS OF SCALE}

To understand the characteristics and interrelationships of analyzing multiple scaled remote sensing data, it is first necessary to define the term "scale." Scale, from a remote sensing perspective, can be defined as the integral (or interval) of space or time over which a measurement is made. The element of scale, therefore, exists as a dimension of observation (i.e., a measurement metric) more so than the dimension of the phenomenon being observed (i.e., the process being observed). Thus, in a multiple scaling approach, the aspect of scale focuses on some unit of measurement that is a defined relationship to some known quantity, such as ground-to-map distance, or a temporal interval (e.g., day, week). From a multiple scaling perspective, the different types of data brought together for analysis will reflect the respective scales at which they were collected, as a function of some defined spatial or temporal measurement characteristic (e.g., $20 \mathrm{~m}$ SPOT multispectral data) (Foody and Curran, 1994).

Two fundamental scales exist to guide the collection, manipulation, and analysis of remote sensing data: (1) space (or spatial) and (2) time (or temporal). Spatial scale, from a practical viewpoint in remote sensing, is governed by the size of the smallest object that can be reliably detected against a spectrally contrasting background. Spatial scale is generally referred to more directly in remote sensing as effective resolution element (ERE). ERE is predicated upon a number of factors, such as instantaneous field of view (IFOV) (i.e., the geometric properties of an imaging system that control sensor resolution characteristics) and other sensor, atmospheric, data processing, and scene properties (Davis and Simonett, 1991). Temporal scale usually denotes the unit of time over which data are collected for analysis (e.g., day, week, year). The time scale of remote sensing data can also refer to a single instant in time or can take on a range of dimensions from historical (before present), current (a time span that envelopes the present within some defined limits), and future.

More generically in remote sensing, space and time scales are embraced within the concept of characteristic scale or scales. Characteristic scale is associated with the recognition and linkage of specific levels of space and time scales by a specific Earth-science discipline. Characteristic scales define the space and time intervals over which a process or processes can be detected or monitored within a specific disciplinary purview (Quattrochi, 1993). For example, atmospheric scientists distinguish microscale versus mesoscale processes as those occurring at spatial scales of 0.01 to $1,000 \mathrm{~m}$ versus 10 to $1,000 \mathrm{~km}$, respectively (Davis et al., 1991). 
These would represent atmospheric characteristics ranging from such phenomena as advection occurring at microscales (e.g., transfer of sensible heat across a small plot of vegetation) to mesoscale or even global advection dynamics related to the movement of weather systems. Characteristic temporal scales for atmospheric scientists may range from a few seconds for determining changes in fluid dynamics of the atmosphere to days in the analysis of hurricanes and attendant atmospheric circulation patterns. Thus, characteristic scales define the space and time intervals with which a process can be detected and monitored; ultimately, characteristic scales influence the type of remote sensing data to use for analysis of specific phenomena in Earth science investigations (e.g., Landsat TM versus AVHRR data to obtain seasonal analysis of vegetation characteristics, vigor, and health).

\subsection{Space and Time Scale Relationships as They Affect TIR Date}

In relation to the use of remote sensing data, specifically to TIR data for the purposes of our discussion here, three overriding questions must be addressed for analysis of the scaling properties of Earth-surface biophysical properties:

- What are the characteristic spatial and temporal scales and scale dependencies of Earth system processes and phenomena?

- What are the measurement scales of remote sensing data and their derived products, and how do these scales vary depending upon sensor calibration and geometric characteristics, data processing algorithms, and correction for the intervening atmosphere?

- How can multiscale remote sensing data, either obtained from the same or different remote sensing systems, be integrated and linked in a statistically robust design to model Earth systems?

These questions are similar to those presented by Turner et al. (1989a) in their concern for predicting across scales in the emerging discipline of Landscape Ecology, and by Davis et al. (1991) in their examination of environmental analysis of remote sensing data. It is our intent to use these questions as a framework for discussing how multiple scaled TIR data can be used to analyze and measure important landscape and biophysical characteristics affecting land-atmosphere interactions.

\subsection{The Influence of Scale Dependence of Earth Surface Varlation and TIR Data}

The observation and measurement of surface processes is highly scale dependent (Ehleringer and Field, 1993). Hence, a phenomenon may appear homogeneous at one spatial scale and heterogeneous at another (Davis et al., 1991). Because spatial heterogeneity constrains the ability to transform information from one scale to another, it is an important factor in integrating remote sensing data ob- 
tained from different scales and from different sensors. For example, Turner et al. $(1989 \mathrm{~b})$ have shown that landscape pattern has a significant influence on the response of measurements to changes in spatial scale. The problems associated with deriving measurements of spatial phenomena from multiple scaled remote sensing data may not occur where homogeneity predominates. Thus, we can also say that in addition to surface phenomena being scale dependent, they are also problem dependent, whereby the stability or variability of the phenomenon or process under observation, as manifested in remote sensing data, will directly influence its qualitative and quantitative interpretation using remote sensing.

Spatial covariability (the degree of scale dependence between values of a spatial process at different locations) is a key consequence of scale dependence. Associated with spatial covariability is the filtering of surface variation across the scene. Because most natural surfaces are highly variable across large areas, surface variation is filtered or regularized across a remote sensing scene (i.e., the surface variation fo natural phenomena is "smoothed" across the scene as a product of sensor IFOV and other sensor-dependent characteristics). Scene regularization is an important feature of remote sensing data (particularly from satellites) that distinguishes these data from other sources of spatial (i.e., geographical) information (Davis and Simonett, 1991). The effects that scene regularization have on remote sensing data can be summarized (from Moik, 1980) by measuring the reflected radiance of a surface at location $x$ at time $t$ :

$$
f(x, \lambda, t, p)=r(x, \lambda, t, p) i(x, \lambda, t)
$$

where $r(x, \lambda, t, p)$ is the reflectance of the surface as a function of position $(x)$, wavelength $(\lambda)$, time $(t)$, and polarization $(p)$, and $i(x, \lambda, t)$ is the incident illumination. This relationship can be simplified by considering only the variation in reflectance with spatial position, $f(x)$.

An important property inherent to scene regularization and variation in remote sensing data is spatial autocorrelation or autocovariance. Spatial autocorrelation measures how $f(x)$ varies as a function of the distance and orientation between observations. If there is any systematic pattern in the spatial distribution of a variable, it is said to be spatially autocorrelated. If the pattern of the phenomenon or process observed via remote sensing is such that nearby or neighboring areas are more similar than more distant areas (with distance referring to spatial separation on the image), then the pattern is positively spatially autocorrelated. Most spatial phenomenon, be it physical (i.e., geomorphological), biophysical (e.g., vegetation type distribution), or cultural exhibits positive spatial autocorrelation patterns. Spatial patterns of Earth-surface phenomena that exhibit negative spatial autocorrelation (i.e., neighboring patterns are not alike) are not common. Between the two extremes are patterns that exhibit no spatial autocorrelation (i.e., random). Spatial autocorrelation is important in remote sensing; the identification, monitoring, measurement, and change in patterns is a fundamental theme in Earth science research. For example, spatial autocorrelation is the basis for the recognition of spatial variability in determining land versus water, field versus forest, or urban versus rural areas (Meentemeyer, 1989). It is often useful in 
remote sensing to search for the level of resolution which maximizes the spatial variability of a phenomenon or process at different spatial and temporal scales. Patterns that have a high degree of positive spatial autocorrelation at one scale of imagery may not have the same degree of spatial autocorrelation at another. Thermal responses for specific phenomenon or processes may be similar (i.e., positively autocorrelated) at a high spatial scale (i.e., high spatial resolution) and may be only minimally autocorrelated at a low spatial scale (i.e., low spatial resolution).

The reception of a signal generated by a particular target will also be affected by the spatial resolution characteristics of the sensing system as they relate to the size of the target (Burnay et al., 1988). For TIR sensors, the radiation from any point in the object is spread out over a small, but finite, area within an image. Consequently, areas with temperatures above their surroundings will appear to have lower temperatures than they actually do, while the converse occurs for areas at a lower temperature than their surroundings. As a result of this effect, large differences between actual temperatures of an object (i.e., those existing for the target being observed at the time of sensing) as measured from TIR data can occur, particularly for small objects (i.e., objects with a small spatial size in reference to the overall scene size of the TIR data) (Burnay et al., 1988). In the case of a multiple scaled TIR remote sensing approach where data acquired from both different sensors and at different spatial resolutions are combined for analysis (e.g., Landsat TM channel $6120 \mathrm{~m}$, and AVHRR $1.1 \mathrm{~km}$ data), this problem would be exacerbated. Although there is no practical way to circumvent this problem, it lends more credence to the concept that from a multiple scaling analysis perspective, the first priority is to know as much information as possible about the overall patterns that comprise the distribution of the phenomena or processes under observation. For example, is it the nature of the target under observation or the underlying processes that form the distribution of the target to be disperse, aggregated, or random? Also, are there any physiographic factors that may have an underlying influence on the thermal response of the phenomena or processes under investigation? These factors (e.g., topography, slope, aspect) would influence the solar illumination of the phenomenon or process and thus, its effective thermal response. Additionally, attributes, such as knowledge of antecedent meteorological events that would possibly influence both the pattern of the target (e.g., precipitation falling over the portion of an AVHRR agricultural scene and not over another) and the thermal response for the target (e.g., excessive soil moisture in one portion of an AVHRR image versus a deficit in another), would affect both the magnitude and distribution of thermal response as recorded by the sensor.

A second priority (at least in most cases) in scaling TIR data is to utilize data from the sensor with the finest spatial resolution (i.e., the highest level of spatial resolution) as a baseline measurement source for understanding the thermal response characteristics of the target. The decision to use a sensor with a high spatial resolution over those with lower spatial resolutions would obviously be affected by the sensor's engineering characteristics, such as the noise-equivalent 
power (NEP). NEP refers to the energy of that input radiation falling on the detector which would give an output signal equal to the noise generated by the detector; the smaller the value of the NEP, the better the detector (Anderson and Wilson, 1984). It can be assumed, however, that a high spatial resolution would lessen any impacts associated with the size of the target, particularly if detection of objects of small size relative to the overall size of the objects in the scene is important. Therefore, as data from sensors with successively lower spatial resolutions are combined with higher resolution TIR data, thermal responses of objects recorded by the sensors for specific ground-resolution cells (GRCs) at the highest possible resolution can be used to "calibrate" the responses recorded at lower spatial resolutions. The GRC is defined as the ground segment sensed at any instant by the IFOV of the sensor. The average value of the spectral reflectance, spectral emissivity, or backscattering cross-section for the GRC, which is recorded as a picture element (pixel), will depend upon the content of the GRC, its heterogeneity, topography, slope, and aspect (Duggin and Robinove, 1990). A linear relationship between TIR values at different spatial scales cannot be assumed; however, use of the highest spatial resolution data as a calibration or reference source will provide some measure to translate thermal response values across spatial scales.

\section{SPATIAL AND TEMPORAL NOISE, IMAGE INFORMATION CONTENT, AND LINEAR AND NON-LINEARITY RELATIONSHIPS IN SCALING TIR DATA}

Noise is the pervasive phenomenon inherent to any remote sensing system. From a classical remote sensing perspective, "noise" can be defined as the introduction of any extraneous signal that competes against the desired signal, and thereby degrades the quality of the remotely sensed data (Colwell, 1983). Noise is usually perceived as being a function of instrument engineering design and performance or signal-to-noise ratio $(\mathrm{S} / \mathrm{N})$, wherein it is most desirable to have the highest ratio of desirable signals to noise. In remote sensing terminology, noise can be classified as coherent (noise signals that have a definite relationship to one another) or random. Sensor noise will obviously have an adverse impact on the derivation of quantitative measurements from multiple scaled TIR data, and its effects may be additive or even multiplicative. Numerous techniques exist, however, to remove the various components of noise from remote sensing data (e.g., Fourier transform) and will not be discussed here (see for example Moik, 1980; Colwell, 1983; Hummer-Miller, 1990).

Also, what may be referred to as Target-to-Sensor (T-S) noise is of critical concern to the analysis of multiple scaled TIR data in Earth science. Here, "noise" is not a function the sensor signal-to-noise ratio, but is a product of the distribution, pattern, arrangement, and overall spatial (and to some extent, temporal) characteristics of biophysical, geographic, and topographic attributes that are manifested as a TIR remote sensing scene. Additionally, the intervening atmosphere between the target and sensor is an important modifier in the interpretation of TIR data. Atmospheric effects as a factor in T-S noise and multiple scaled TIR 
data will be discussed in Section 5. Figure 1 illustrates how the smallest viewed ground element by a specific sensor, or GRC, can be affected by T-S noise. Dependent upon the overall composite of GRCs or "footprint" inherent to a sensing system, a TIR scene is influenced by the heterogeneity of the biophysical, topographic, and geographic parameters being sensed. For example, TIR data obtained from an airborne platform with a high spatial resolution (e.g., $10 \mathrm{~m}$ ) will be much more sensitive to higher T-S frequencies (i.e., distinct changes in biophysical or ground characteristics at microscales) than similar data acquired from sensors with lower spatial resolutions from the Landsat TM or AVHRR satellites. Thus, heterogeneity at the surface will be manifested as various frequencies of T-S noise as a function of the smallest viewed ground element of the sensor. This issue is further extended in Figure 2, wherein the effects of the atmosphere modify the thermal radiance upwelling from the ground and reaching the sensor, which modulates T-S noise.

In the use of TIR for measurement of surface thermal attributes or processes, some fundamental principals of image acquisition or analysis are: (1) the radiance properties recorded in the image accurately represent both the thermal properties of the phenomenon or process (i.e., target) being recorded; (2) the imaged data accurately represent the relative spatial (and temporal) frequency of the phenomenon or process as it exists on the ground within the limits of the GRC resolving capabilities of the imaging system; and (3) after processing, the image data derived from TIR sensors can be rectified and superimposed on a map to represent accurately the ground features under observation (Duggin and Robinov, 1990). Points (1) and (3) are intimately related to the engineering characteristics of the sensing system and the software used to process the data. Although these points are obviously important to any discussion on multiple scaling of TIR data, we wish to elaborate more upon point (2) and its relevance to both scale dependency and the affect of integrating data collected at different measurement scales.

\subsection{Scene Versus Sensor Spatial Frequency}

The spatial frequency of discernible variations in radiance from each part of the scene will depend upon the thermal contrast between scene elements and on built-in limitations of the sensor (i.e., engineering characteristics). A remote sensing scene will have a two-dimensional spatial frequency spectrum (power spectrum) that is related to the spatial variation in upwelling thermal radiance levels across the area in the scene. Each pixel in the image results from the averaging of the upwelling thermal radiance within each GRC after modulation by the atmosphere and the sensing system (Figures 1 and 2). The two-dimensional scene spatial frequency is delineated in terms of pairs of pixels within the sampled image (Duggin and Robinov, 1990).

Within the concept of scene frequency, it is assumed there is a high degree of correlation between the surface attributes of the target, the thermo-optical properties of the ground cover (i.e., the thermal radiance characteristics of the ground 


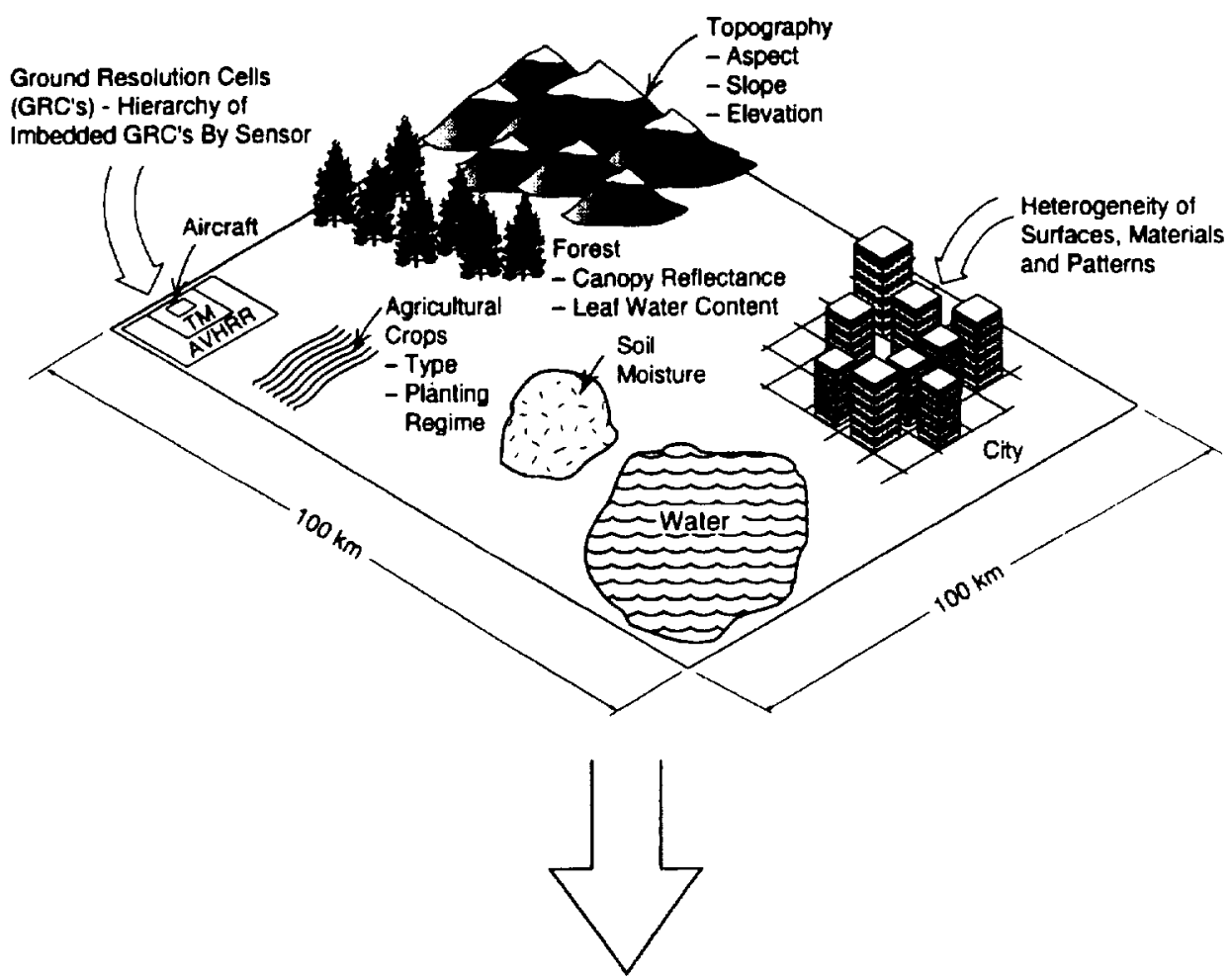

Biophysical

Topography

Geographic Pattern

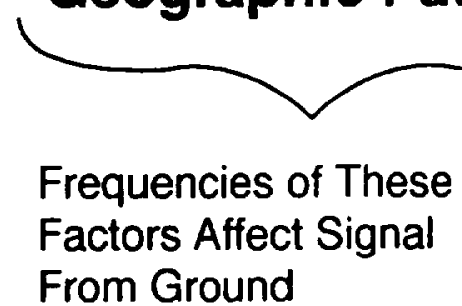

FIGURE 1 Illustration of the relationships between Ground Resolution Cells (GRCs) and differing frequencies of biophysical, topographic, and geographic pattern as they affect Target-to-Sensor (T-S) noise. Heterogeneity at the surface will be manifested as various frequencies of T-S noisc, depending upon the content of the GRC as recorded by the sensor.

cover as related to its biophysical properties), and the attributes of the acquired image (e.g., radiometric quality). For TIR data, relations between the variables giving rise to an upwelling spectral radiance response from a heterogeneous assemblage of scene components at ground level and radiance levels recorded for each GRC may be expressed as follows (Duggin and Robinov, 1990): 

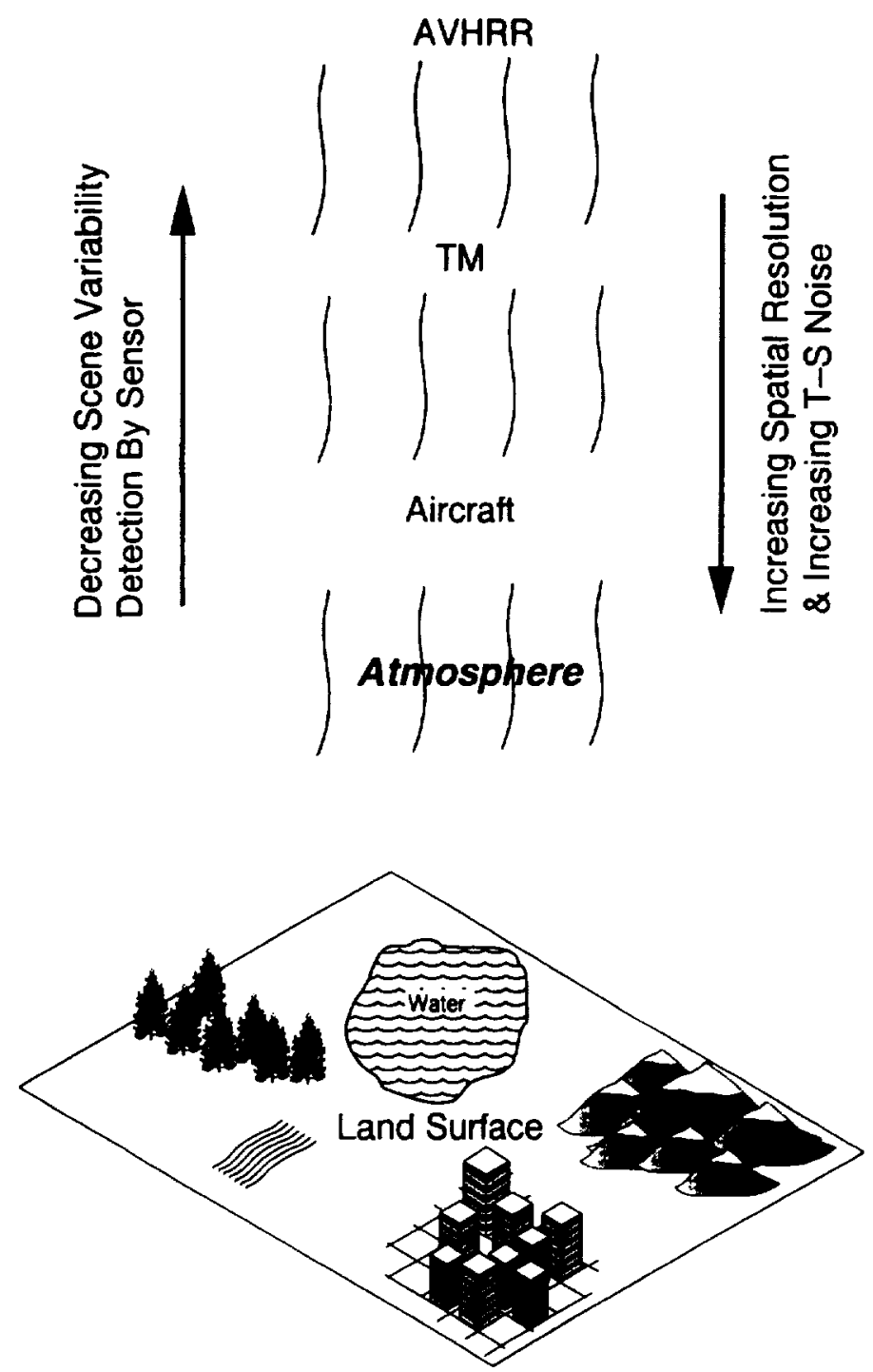

FIGURE 2 Illustration of the relationships between surface heterogeneity and T-S noise sensitivity as modified by the atmospherc.

$$
\begin{aligned}
L_{(i, j)}= & \frac{1}{b_{i, j}} \sum_{l=1}^{l=n} \int_{f_{1}}^{f_{2}} \int_{\lambda_{1}}^{\lambda_{2}} I(\lambda, f) \tau\left(\theta^{\prime}, \phi^{\prime}, \lambda\right) \int_{x_{1}}^{x_{2}} \int_{y_{1}}^{y_{2}} W_{l}(T) g_{l}(X, Y) \epsilon_{l}\left(\theta^{\prime}, \phi^{\prime}, \lambda\right) \\
& \times \xi(X, Y ; x, y, \lambda) d Y d X d \lambda d f+\int_{f_{1}}^{f_{2}} \int_{\lambda_{1}}^{\lambda_{2}} L_{\text {self }}\left(\theta^{\prime}, \phi^{\prime}, \lambda\right) I(\lambda, f) d \lambda d f-a_{i, j}
\end{aligned}
$$


where

$$
\begin{aligned}
& L_{(i, j)}= \text { calibrated radiance value for pixel }(i, j) \\
& 1 / b_{i, j}= \text { gain of sensor for pixel }(i, j) \text {. This may vary slightly from } \\
& \text { element to element of a focal plane array detector } \\
& \tau\left(\theta^{\prime}, \phi^{\prime}, \lambda\right)= \text { spectral transmission of atmosphere along path to sensor with } \\
& \text { zenith angle } \theta^{\prime} \text { and azimuth } \phi^{\prime} \\
& g_{l}(X, Y)= \text { delta function which is equal to } 1.0 \text { is scene element } l \text { is } \\
& \text { present in GRC }(i, j) \text { at position }(X, Y) . \text { The fraction of the } \\
& \text { GRC filled by scene element } l \text { is } \\
& \qquad \int_{X_{1}}^{X_{2}} \int_{Y_{1}}^{Y_{2}} g_{l}(X, Y) d Y d X \\
& \qquad \sum_{l=1}^{l=n} \int_{X_{1}}^{X_{2}} \int_{Y_{1}}^{Y_{2}} g_{l}(X, Y) d Y d X \\
& a_{i, j}= \text { offset of sensor for pixel }(i, j) \text {. This may vary slightly from } \\
& \text { element to element of a focal-plane array detector } \\
& I(\lambda, f)= \text { spectral response of sensor for electronic frequency } \\
& W_{l}(T)= \text { Black-body radiant exitance for scene element } l \text { at absolute } \\
& \text { temperature } T \\
& \epsilon_{l}\left(\theta^{\prime}, \phi^{\prime}, \lambda\right)= \text { spectral emissivity of scene element } l \text {, viewed with zenith an- } \\
& \text { gle } \theta^{\prime} \text { and azimuth angle } \phi^{\prime} \\
& L_{\text {self }}\left(\theta^{\prime}, \phi^{\prime}, \lambda\right)= \text { spectral self-radiance of the atmosphere viewed from zenith } \\
& \text { angle } \theta^{\prime} \text { and azimuth angle } \phi^{\prime} \\
& \xi(X, Y ; x, y, \lambda)= \text { the point-spread function }(\text { PSF) of the sensor at wavelength } \\
& \lambda, \text { for radiance from ground coordinates }(X, Y) \text {, producing a } \\
& \text { point-like image at point }(x, y) \text { on the focal plane }
\end{aligned}
$$

where there are $n$ scene elements in the GRC.

It is obvious from equation (2) that the spatial attributes (i.e., homogeneity or heterogeneity) of the ground-cover types included in the GRC will affect the intensity of thermal radiance from the ground and the spatial distribution of upwelling radiance signatures reaching the TIR remote sensing system. Thus, the spatial frequencies of the phenomenon or process under investigation will ultimately be translated into a high or low sensor spatial frequency relationship (i.e., $\mathrm{T}$-S noise factor) as recorded by the sensor. For the spatial frequency domain $(\mathcal{F})$ of the TIR sensor then,

$$
\mathcal{F}\{I(x, y)\}=E\left(\omega_{x}, \omega_{y}\right)
$$

where $(x, y)$ are spatial coordinates of a location on the focal plane and $\left(\omega_{x}, \omega_{y}\right)$ are the spatial frequencies recorded in the image plane in the $x$ and $y$ directions. The sampled power spectrum is represented by $E\left(\omega_{x}, \omega_{y}\right)$. It is the power spectrum of the image with spatial frequency expressed in pixel pairs (i.e., GRC pairs) and is a sample of the power spectrum of the radiance field upwelling from the surface. Also,

$$
\mathcal{F}\{L(X, Y)\}=F\left(\omega_{x}, \omega_{y}\right)
$$


where $F\left(\omega_{x}, \omega_{y}\right)$ is the power spectrum of the radiance upwelling from the object space or plane on the ground and where $\left(\omega_{x}, \omega_{y}\right)$ are spatial frequencies in the $X$ and $Y$ directions in object space. The power spectrum of the upwelling radiance field is continuous and will be influenced by the following factors (Duggin and Robinov, 1990):

1. heterogeneity of the scene;

2. nature of the scene elements (i.e., heterogeneity and homogeneity);

3. illumination and view-angle geometry of the scene;

4. shape, size, and arrangement (pattern) of the scene elements (i.e., target characteristics) within the TIR wavebound(s) used;

5. scale: size and shape of the GRC (i.e., the spatial resolution of the GRC as a function of the IFOV of the sensor and the variation of the sensor response across the GRC);

6. topographic variations (slope, aspect, elevation) within the GRC and the interaction of such variations with scene elements and their shape, size, and pattern as they may be affected by illumination and view-angle geometry;

7. atmospheric effects (path radiance and transmittance); and

8. degradations in radiometric accuracy between pixels arising from sensor engineering and calibration errors (e.g., sensor noise).

The effects noted above (and illustrated in Figures 1 and 2) may be additive, multiplicative, or both and, as a consequence, may have pronounced influence on T-S noise ratios. Scene versus spatial frequencies are also time dependent. The eight items listed above may be highly influenced by temporal changes; thus, comparing differences in images over time will be affected by corresponding changes in the target (e.g., landscape changes, vegetation canopy, soil moisture), the intervening atmosphere (e.g., humidity, temperature, barometric pressure), and in sensor radiometric characteristics (e.g., variations in gains and offsets that influence sensor radiometric fidelity).

\subsection{Image Information Content and Non-Linearitles In Surface Temperatures in Multiple Scaled TIR Data}

Spatial and temporal frequency differences also reflect on the overall information content or entropy of the scene. The entropy of a probability distribution may be used as a measure of the information content of a symbol (i.e., image) selected from this distribution. For an image, the entropy $H_{e}$ of $n$ random variables with probability distribution $p_{k}$ is defined as

$$
H_{e}=-\sum_{k=1}^{n} p_{k} \log _{2}\left(p_{k}\right)
$$

where $\log _{2}$ has a unit of information in bits (Moik, 1980). As $H_{e}$ increases, so does the randomness of information content of the image. Images with high entropy values in the case of TIR data are hard to deal with. As entropy increases, 
it becomes increasingly difficult to derive accurate surface temperatures because the scene variability on a per pixel basis exceeds the ability of the sensing system to detect relative changes in surface temperatures between pixels. Thus, as entropy increases, there is a corresponding increase in the non-linearity in surface temperatures as measured by remote sensing systems. This problem must be considered in attempting to relate temperature differences between TIR data collected at multiple scales. Here the scale at which the data are collected (as well as sensor engineering characteristics) will influence the amount of entropy extant in the scene. This will influence the derivation of surface temperatures from a particular scene. Comparison of surface temperatures obtained from TIR data collected over heterogeneous landscapes and at high spatial resolutions (i.e., large spatial scales) with TIR data over the same area acquired at lower spatial resolutions (i.e., small spatial scales) will be subject to the vagaries of entropy differences. It is most important, therefore, to be aware of the spatial frequencies inherent to the phenomenon or process (or landscape) under investigation in attempting to correlate derived surface temperatures from multiple scaled TIR data.

Compounding the problems related to entropy maximization between multiple scaled TIR data is the factor of the Stephan-Boltzman equation

$$
W=\sigma T^{4}
$$

where $W$ is radiant emittance in $\mathrm{W} \mathrm{m}^{-2}, \sigma$ is the Stephan-Boltzman constant (5.6697 $\times 10^{-8} \mathrm{~W} \mathrm{~m}^{-2}{ }^{\circ} \mathrm{K}^{-4}$ ), and $T$ is absolute temperature in ${ }^{\circ} \mathrm{K}$. Because it takes only a small change in energy to effect a large change in temperature, comparison of surface temperatures from multiple scaled TIR data will be highly influenced by scene entropy as recorded by the sensor, along with the T-S noise factors listed in Section 3.1. This stresses the need to obtain data with known radiometric calibration coefficients so that the calculation of surface temperatures will be as precise as possible within the limits of sensor engineering characteristics. Otherwise, the derivation of surface temperatures from multiple scaled TIR data (obtained from both the same and different sensors) and the comparison of these temperatures at their respective scales will provide highly questionable results.

An allied factor that may contribute to non-linearities in surface temperatures as recorded by multiple scaled TIR sensors is that of the angular reflectance characteristics of the target or targets under consideration. Angular reflectance, as a result of both the geometry of the target (e.g., uneven forest or vegetation canopy, differences in pitch angles of rooftops) and sensor-to-target geometric relationships (i.e., off-nadir view angles) are much more pernicious in the visible and reflective IR wavelengths as opposed to TIR data. Surface non-uniformity, however, may influence temperature measurements from thermal data. The intricacies of Lambertian and non-Lambertian surfaces (i.e., uniform and nonuniform) and their relationships to remote sensing have been explored in-depth (see, for example, Gerstl and Simmer, 1986; Kaufman, 1982, 1984, 1989) and they will not be discussed in detail here. It is important to relate, however, that the maximum amount of energy is emitted (i.e., radiated) by a body perpendicular to 
its surface plane. Conversely, no TIR energy is radiated tangentially to a body's surface. Targets emit uniform amounts of TIR energy within a $30^{\circ}$ boundary from the plane perpendicular to the surface plane (Baraniak, 1983). Beyond this $30^{\circ}$ boundary, there is an edge effect that occurs as a result of the Cosine Law falloff. This results in a reduction of energy received by a sensor within its IFOV. For example, if a highly polished spherical or circular surface is viewed by a TIR sensor, edges of the objects imaged will appear different from their centers. This effect is continual for rough-textured surfaces and thus, a highly diffusing, rough surface is referred to as a Lambertian emitter (Baraniak, 1983). It behaves according to Lambert's Law which states that the incident energy from a target to a detector (i.e., sensor) is equal to the amount of energy times the cosine of the angle of emittance. Many natural bodies are approximately full radiators (i.e., Lambertian emitters), but the effects of non-Lambertian radiators cannot be discounted, particularly in using multispectral TIR data. The effects of Lambertian versus non-Lambertian surfaces on TIR data collected at multiple spatial scales by different sensing systems have not been examined. It may be hypothesized, however, that the Lambertian or non-Lambertian characteristics of a target under consideration would be effected by the scales of observation and the homogeneity or heterogeneity of the surface. Thus, as the spatial scale of TIR data becomes smaller (i.e., from $5 \mathrm{~m}$ to $1 \mathrm{~km}$ pixel size), there would be a greater probability of surfaces to assume more Lambertian characteristics because the thermal responses become more aggregated, and the cosine of the angle of emittance becomes less of a significant factor in the amount of energy recorded by a sensing system. The non-Lambertian effects caused by the heterogeneity in pattern of a surface would be diminished as the spatial scale of observation using a TIR sensor becomes smaller. For example, the non-Lambertian effects associated with a mixed forest-cropland landscape would become less significant as the spatial scale of observation decreases from $10 \mathrm{~m}$ to $30 \mathrm{~m}$ because the scene frequencies become more homogeneous. There may also be a temporal dimension associated with this effect, particularly in regard to the study of Earth-surface processes that are dynamic and change through time and seasons. Again, the relationships of Lambertian versus non-Lambertian emittance as a function of scale remain to be fully examined, but they should be considered within the context of relating data obtained at multiple space and time scales for analysis of landscape and biophysical processes using TIR remote sensing.

\subsection{Scale Invariance and Variance in Multiple Scaled TIR Data}

A major concern arising from the use of data acquired either from the same TIR sensor at different scales, or from different TIR sensors with varying spatial resolutions, is whether thermal response measurements vary or are invariant with scale. Although this topic has been alluded to in the previous sections, it is useful to explore further the factor of scale variance or invariance in relating surface temperatures because of its overall importance to analysis of multiple scaled TIR data. Most TIR sensors are designed so that the effective spectral radiance 
TABLE 1

Comparison of TIR Remote Sensing Systems That Have Been Used to Derive Thermal Radiances for Landscape Processes

\begin{tabular}{|c|c|c|c|c|}
\hline $\begin{array}{l}\text { Imaging } \\
\text { System }\end{array}$ & $\begin{array}{l}\text { Spectral } \\
\text { Response } \\
(\mu \mathrm{m})\end{array}$ & $\begin{array}{l}\text { IFOV } \\
\text { (mR) or } \\
\text { Ground } \\
\text { Spot Sizc } \\
(\mathrm{km})\end{array}$ & $\begin{array}{l}\text { Noise } \\
\text { Level } \\
\left({ }^{\circ} \mathrm{K}\right)\end{array}$ & $\begin{array}{c}\text { Data } \\
\text { Quantization } \\
\text { (bits) }\end{array}$ \\
\hline $\begin{array}{l}\text { Landsat Thematic } \\
\text { Mapper (Band 6) } \\
\text { (TM) }\end{array}$ & $10.4-12.4$ & $0.12 \mathrm{~km}$ & $0.4 \mathrm{~K}$ & 8 \\
\hline $\begin{array}{l}\text { Hcat Capacity } \\
\text { Mapping Mission } \\
\text { (HCMM) }\end{array}$ & $10.5-12.5$ & $0.83 \mathrm{mR}$ & $0.4 \mathrm{~K}$ & 8 \\
\hline $\begin{array}{l}\text { Advanced Very High } \\
\text { Resolution } \\
\text { Radiometer } \\
\text { (AVHRR) }\end{array}$ & $\begin{array}{l}3.55-3.93 \\
10.3-11.3 \\
11.5-12.5\end{array}$ & $\begin{array}{l}1.51 \mathrm{mR} \\
1.41 \mathrm{mR} \\
1.30 \mathrm{mR}\end{array}$ & $0.12 \mathrm{~K}$ & 10 \\
\hline $\begin{array}{l}\text { Thermal Infrared } \\
\text { Multispectral } \\
\text { Scanner } \\
\text { (TIMS) }\end{array}$ & $\begin{array}{l}8.2-8.54 \\
8.6-8.95 \\
9.01-9.35 \\
9.40-10.15 \\
10.2-11.2 \\
11.26-11.60\end{array}$ & $2.5 \mathrm{mR}$ & $0.1-.3 \mathrm{~K}$ & 8 \\
\hline
\end{tabular}

(Adapted from Schott, 1989, p. 1311.)

observed can be be expressed as

$$
L_{\lambda}=\epsilon_{\lambda} L_{T \lambda} \tau_{\lambda}+r_{\lambda} L_{d \lambda} \tau_{\lambda}+L_{\mu \lambda}
$$

where

$L_{\lambda}=$ the observed spectral radiance in $\mathrm{Wm}^{-2} \mathrm{sr}^{-1} \mu \mathrm{m}^{-1}$

$\epsilon_{\lambda}=$ the wavelength dependent emissivity

$L_{T \lambda}=$ is the spectral radiance from a blackbody at temperature $T$ according to Planck's equation

$\tau_{\lambda}=$ is the wavelength dependent atmospheric transmission

$L_{d \lambda}=$ is the spectral downwelled radiance onto the target due to self-emission by the sky

$r_{\lambda}=$ is the wavelength dependent reflectivity

$L_{\mu \lambda}=$ is the spectral upwelled radiance due to self-emission from the atmosphere between the target and the sensor

$\lambda=$ wavelength (Schott, 1989).

In discussing potential problems associated with scale variance or invariance, it is useful to compare selected features of several TIR sensing systems used to derive thermal radiance values for landscape phenomena or processes. These features are presented in Table 1 . The amount of radiance recorded by a par- 
ticular sensor as expressed in equation (7) is modified by the components of equation (2) and the power spectrum factors noted in Section 3.1. If thermal radiance is variant with scale, then TIR data obtained at multiple space (and time) scales would be very useful for analyzing the physical characteristics of the target under observation (e.g., biophysical attributes, pattern and arrangement) and the thermophysical influences at the surface (e.g., aerodynamic roughness, wind). Thus, the real virtue of using TIR data obtained at different spatial and temporal scales would be to assess how effective surface temperatures are manifested in multiscaled TIR images obtained over the same area on the Earth's surface. Detection of variations in temperatures with scale from TIR sensors would permit more accurate modeling of landscape energy fluxes for developing a better understanding of how thermal energy is partitioned across landscapes and how these energy fluxes influence, or force, changes in land-atmosphere interactions at local, regional, and global scales. Measurement of variations in effective radiometric temperatures from multiple scaled TIR data would provide insight into how energy fluxes and landscape processes are related. Inherent within this is whether TIR data obtained at regional scales (e.g., AVHRR $1.1 \mathrm{~km}$ resolution) can be used to predict or model thermal energy flux relationships at larger (i.e., finer) spatial scales. The factor of scale variance in thermal responses would be critical to understanding whether surface temperatures could be predicted across scales, either from a "bottom-up" (i.e., larger to smaller spatial scales) or "topdown" (i.e., smaller to larger scales) approach. This will be examined more fully in the discussion on modeling and multiple scaled TIR data in Section 6.

Because of the paucity of references on multiple scaling of TIR data, there is only limited evidence on the interrelationships of thermal responses recorded at different spatial scales. The NASA FIFE project conducted over the Konza Prairie in central Kansas has produced results that are inconclusive in defining whether surface thermal energy responses are variant or invariant, as recorded by different remote sensing systems and at different spatial scales. In a summary research article, Hall et al. (1992) describe their results in attempting to understand whether surface temperatures over the FIFE site were variant or invariant with spatial scale. Data from the Landsat TM thermal band $\left(120 \mathrm{~m}^{2}\right.$ spatial resolution) were used to calculate temperature in two ways: (1) at the $120 \mathrm{~m}$ "patch" level, using TM measured radiance and then averaging the $120 \mathrm{~m}$ results to obtain a mesoscale value of $T\left({ }^{\circ} \mathrm{K}\right)$ at $1 \mathrm{~km}$ (to approximate the $1.1 \mathrm{~km}^{2}$ pixel size of AVHRR data); and (2) by calculating $T$ directly at the $1 \mathrm{~km}$ level by aggregating the TM radiance to $1 \mathrm{~km}$, and then applying the standard relationship between radiometric temperature and emittance $X$ given for the Landsat TM thermal band (from Markham and Barker, 1986) as

$$
T=K_{2} / \ln \left(K_{1} / X+1\right)
$$

where $X$ is spectral radiance in $\mathrm{mW} \mathrm{cm} \mathrm{cm}^{-2} \mathrm{~s}^{-1} \mu \mathrm{m}^{-1} ; K_{1}=67.2 \mathrm{~mW} \mathrm{~cm} \mathrm{sr}^{-1}$ $\mu \mathrm{m}^{-1}$; and $K_{2}=1284.3^{\circ} \mathrm{K}$. Hall et al. (1992) assumed this relationship for the range of temperatures between $27^{\circ} \mathrm{C}-37^{\circ} \mathrm{C}$ that commonly occurred during the growing season over the FIFE site. They found good agreement in using TM 
thermal data to approximate changes in spatial scale from $120 \mathrm{~m}$ to $1 \mathrm{~km}$ levels; i.e., surface temperature in this experiment was invariant with spatial scale. They do note, however, that approximating how surface energy fluxes (e.g., sensible heat) change with scale using satellite data is problematic.

In a related study from the FIFE program, Goetz et al. (1993, 1994) compared thermal responses as recorded by a helicopter-mounted Modular Multispectral Radiometer (MMR) (25 $\mathrm{m}^{2}$ spatial resolution), the NS001 Thematic Mapper Simulator (TMS) flown onboard on C-130 aircraft $\left(400 \mathrm{~m}^{2}\right.$ spatial resolution), and from the Landsat $5 \mathrm{TM}\left(120 \mathrm{~m}^{2}\right.$ spatial resolution). They found that intercomparison of TM thermal data with near-surface level temperature measurements ranged from $1{ }^{\circ} \mathrm{C}$ to $8^{\circ} \mathrm{C}$, with the Landsat TM consistently overestimating surface temperature (a result primarily of the TM sensor radiometric characteristics). In contrast, surface thermal temperatures recorded by the MMR and NS001 sensors for the same site, were in reasonable agreement with near-surface level temperatures, with standard errors of $2.0^{\circ} \mathrm{C}$ and $2.2{ }^{\circ} \mathrm{C}$ for vegetated targets.

Obviously, the question of whether surface temperatures as manifested in multiple scaled remote sensing data are variant or invariant with spatial scale is open for more thorough measurement and research. The development of a more quantitative insight into how, or if, surface temperature changes with scale is critical to understanding whether there are any thresholds that must be dealt with in attempting to model surface temperatures at small spatial (i.e., broader) scales using remote sensing. This is particularly acute in refining global climate change models using Global Circulation Models (GCMs). For example, Garratt et al. (1993) found that GCMs tend to overestimate the mean monthly levels of net radiation by about $15 \%-20 \%$ on an annual basis for observed annual values in the range of 50 to $100 \mathrm{Wm}^{-2}$. This results from several deficiencies in modeling, including land-surface temperatures being underestimated resulting in underestimates of the outgoing longwave flux. Thus, it is important to identify any breaks or thresholds in longwave flux (i.e., upwelling longwave energy from the Earth's surface) from local to regional scales that may contribute to errors in net radiation estimates using GCMs.

\section{NEIGHBORHOOD CONSTRAINTS ON MULTIPLE SCALED TIR DATA}

Another factor that affects the analysis (and modeling) of TIR data for measurement of thermal responses of landscape phenomena is the aspect of neighborhood constraints. These constraints can be generalized into two types: (1) Bayesian or decision-making; and (2) time dependent. Bayesian constraints relate to the restrictions placed on the use of TIR data in using the results from remote sensing data to infer (or predict) otherwise unknown surface temperatures. Thus, instead of predicting actual surface temperatures, remote sensing data are used as a surrogate for observed temperatures on the ground. For example, it cannot be assumed that a set of thermal responses for a specific landscape phenomenon or process measured using a specific TIR sensor (e.g., AVHRR) can be used to 
predict the same TIR measurements either from other sensors, or from images recorded at different times using the same sensor. Bayesian constraints are a product of factors affecting upwelling longwave radiance from the surface [equation (2)] and from those associated with scene versus sensor spatial frequency listed in Section 3.1. We cannot, therefore, have clear a priori knowledge (i.e., as inferred from the imagery) of which of the many landscape, biophysical, energy flux, and sensor engineering parameters will affect the inferential measurement of temperature from TIR remote sensing data. Sensitivity studies and thermophysical considerations of the target under observation, the structure of the intervening atmosphere, and the sensing system used should provide some guidance toward developing better a priori assessments of the constraints that may be placed on the variety of surface temperature measurements from TIR data. Although there is a priori uncertainty in predicting surface temperatures from TIR data [see Price (1985) for a discussion of some the a priori considerations that must be made], further examination fo TIR data for analyses of land-surface and land-atmosphere interactions should, from a Bayesian perspective, improve the predictability of TIR measurements. This is particularly true in improving confidence in predicting how, or if, surface temperatures change over various landscapes using a multiple scaled TIR remote sensing approach.

The second neighborhood constraint on using TIR data is time. A number of studies using TIR data show that time-dependent relationships are good indicators of surface temperature changes measured from multitemporal remote sensing data (Lynn, 1986). Data from the Heat Capacity Mapping Mission (HCMM), for example, have been used extensively for measurement of changes in surface temperatures through time (Short and Stuart 1982; Carlson, 1986). Additionally, much work has been done applying TIR remote sensing data to obtain estimates of thermal inertia, which is a measure of the ability of a material to resist change in temperature. Thermal inertia is a physical property of the surface material, equal to $(K \rho c)^{1 / 2}$, where $K=$ thermal conductivity, $\rho=$ density, and $c=$ specific heat (see for example Kahle et al., 1984; Kahle and Alley, 1985; Kahle, 1987). Although the factor of temporal dependency in multiple scaled TIR data has been alluded to in Section 2, time can also be examined as a neighborhood constraint; i.e., the changes in $\Delta T$ for the same and neighboring phenomena as represented by TIR remote sensing cannot exceed certain reasonable limits. Time constraints result from the frequency of data collection (i.e., the time span between which data are collected), and the sensor engineering characteristics of the same and different sensors. For example, TIMS data have been used to measure short-term thermal responses in forested landscapes as an estimate of canopy heat storage, which is important in determining sensible and latent heat fluxes for forests (Luvall and Holbo, 1989; Luvall et al., 1990). Although the magnitude of change in observed temperatures for selected forested landscapes depends on tree type (e.g., pine plantation, natural forested environment, clearcut or burned area), these measurements should not exceed some reasonable temperature, given a relatively short time span (15-30 minutes) between TIMS data collection over the same site. One would assume, therefore, that a $\Delta T$ greater than say, $5^{\circ} \mathrm{C}-7^{\circ} \mathrm{C}$ calculated from TIR data for a homogeneous forested landscape within a 15-30 
minute period, would be unreasonable. Microclimatic factors, such as soil moisture conditions, overshadowing, wind eddying, and surfaces being held around freezing, will undoubtedly have an effect on temporal changes in predicted surface temperatures using TIR data, but the resulting predicted surface temperatures should be within some reasonable dynamic range. Obviously, the problem of time constraints will be exacerbated in using multiple scaled TIR data, and an a priori knowledge of the space and time factors that influence the upwelling longwave radiance recorded by TIR remote sensors will be required to derive accurate surface temperature measurements.

\section{ATMOSPHERIC EFFECTS AND THEIR INFLUENCE ON MULTIPLE SCALED TIR DATA}

The prediction of surface temperature is a function of the heat-flow equation, which describes the behavior of temperature $(T)$ in a solid, such as rock or soil. The heat-flow equation relates observed surface temperatures to the energy flux exchanged with the atmosphere and is given by

$$
p c \frac{\partial T}{\partial t}=\frac{\partial}{\partial z}\left(k \frac{\partial T}{\partial z}\right)
$$

where temperature is a function of depth $z$, measured downward from the surface, and time $t, p$ is the density $\left(\mathrm{kg} \mathrm{m}^{-3}\right), c$ is the heat capacity $\left(\mathrm{J} \mathrm{kg}^{-1}\right)$, and $k$ is the thermal conductivity $\left(\mathrm{J} \mathrm{m}^{-1} \mathrm{sec}^{-1}\right)$. For remote sensing applications, equation (9) is solved to yield $T$ at the Earth's surface. The solution to equation (9) assumes a surface boundary condition, which may be provided either by specification of the value of temperature as a function of time at the surface $z=0$, or by a condition placed on the surface energy flux (Price, 1989). This condition can be given by specifying the value of the ground heat flux $G$ at the surface, where

$$
G(z, t)=-k \frac{\partial T}{\partial z}
$$

Predicting surface temperatures using remote sensing employs an inverse solution to the heat-flux equation; i.e., the thermal radiance of the target in question is recorded by the sensing system, the inferred temperature is derived via Planck's equation, and a thermophysical understanding of the derived temperatures is sought that is consistent with the temperature value that is measured. As noted earlier, emissivity is an important factor that must be considered in accurately predicting the surface temperature of objects on the ground from remote sensing. Although it will not be discussed here, calculation of emissivities for ground objects from remote sensing has been measured using different techniques that are applicable to accurately deriving the surface temperatures of a variety of Earth surfaces (see, for example, Artis and Carnahan, 1982; Kahle, 1987; Hook and Kahle, 1990).

Outside of emissivity (and besides sensor radiometric engineering and calibration characteristics), the most important influences on deriving accurate surface 


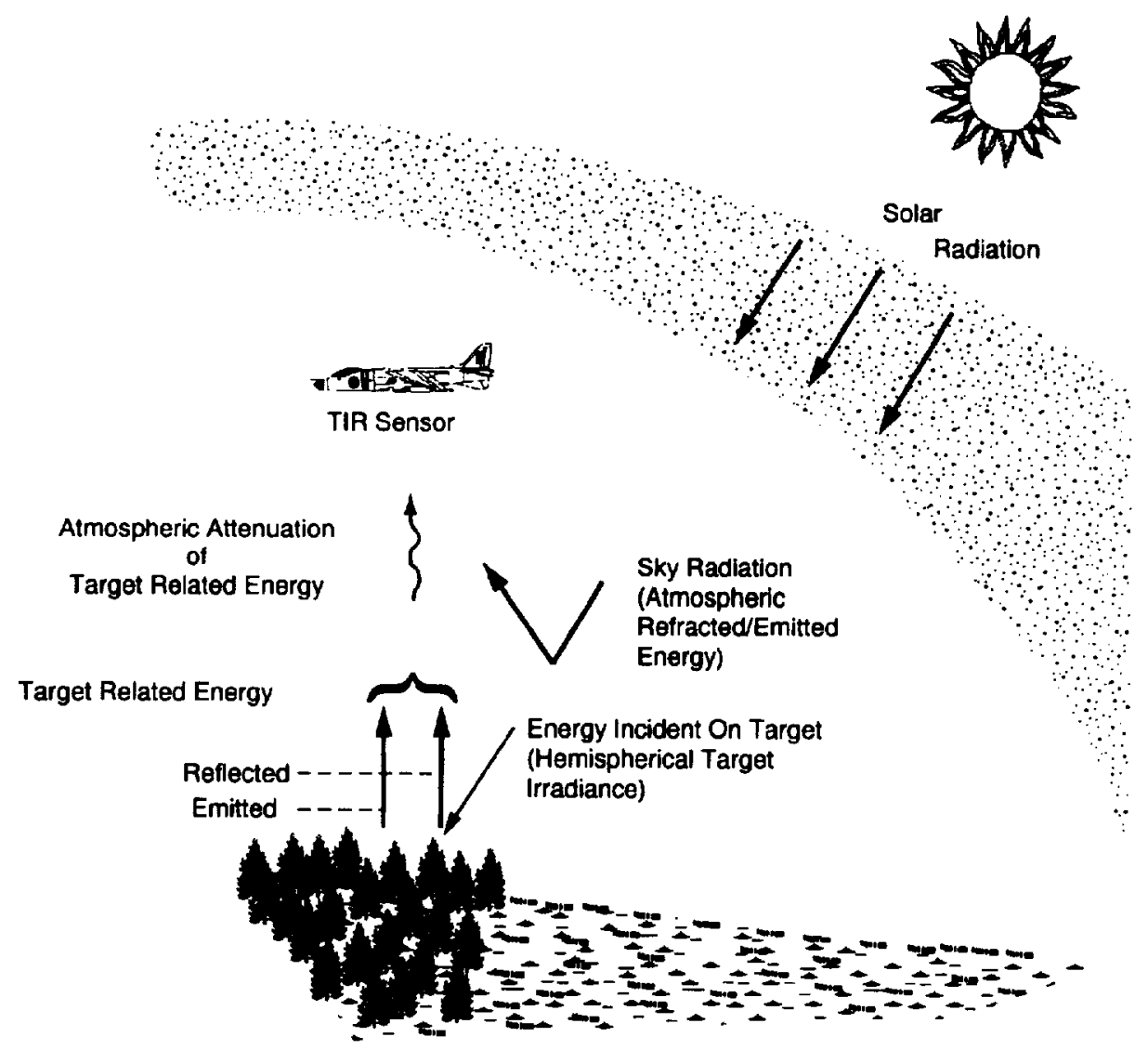

FIGURE 3 Overview of the components of total thermal IR radiation upwelling from the Earth's surface that reaches a remote sensing platform.

temperatures from TIR remote sensing data are the effects of the intervening atmosphere between the sensor and the ground. Because of their importance in predicting surface temperatures, atmospheric effects need to be examined to understand how they can be mitigated using atmospheric correction measures and to speculate on how they will be exacerbated when using multiple scaled TIR data. The atmosphere influences the interpretation of TIR data in three ways: (1) the effect of sky radiation falling on the surface, (2) the absorption of IR radiation by the atmosphere (atmospheric path transmission), and (3) the contribution of emission by the atmosphere to the radiation received (atmospheric radiance) (Anderson and Wilson, 1984) (Figure 3). The contribution of atmospheric scattering to the received radiation may be disregarded as a major component of atmospheric effects, since this is negligible for TIR wavelengths.

The quantity of radiation from the sky incident on the surface depends on the low-level air temperature and on the amount of cloud cover; humidity and gaseous composition have smaller effects. The amount of radiation from a clear 
sky is calculated by

$$
L_{\text {sky }}=-170.9+1.195 \sigma T_{A}^{4}
$$

where $L_{\text {sky }}$ is measured in $\mathrm{Wm}^{-2}$, Stefans constant $\sigma=5.660 \times 10^{-8} \mathrm{Wm}^{-2} \mathrm{~K}^{-4}$, and $T_{A}$ is air temperature in ${ }^{\circ} \mathrm{K}$. Using equation (11), it is possible to accurately calculate the downward sky radiation flux. The measurement of sky radiation can be carried out either by having an upward-pointing radiometer (e.g., pyrgeometer) at the surface or by placing a large sheet of material with high reflectivity, such as aluminum, on or near the surface. When viewing this sheet, a TIR scanner will see mainly reflected sky radiation. If the temperature and emissivity of the sheet are known, the sky radiation can be assessed exactly (Anderson and Wilson, 1984).

Two other factors associated with atmospheric effects on TIR data are more difficult to accurately determine. Atmospheric absorption influences the quantity and quality of thermal energy reaching the sensor. For data collection with aircraft-mounted sensors, only the effects of water vapor and aerosols need be dealt with. For satellite data, however, the effects of other absorbers that exist higher in the atmosphere must be considered, such as ozone and carbon dioxide. Aerosols are particularly vexing to account for, since it is difficult to determine local atmospheric concentrations. This problem is usually overcome by conducting TIR airborne surveys when optical visibility is high (i.e., $>25 \mathrm{~km}$ ) because there is a close relationship between optical visibility and aerosol concentration (Lee, 1973; Anderson and Wilson, 1984). The existence of water vapor cannot be handled in a similar manner and it is necessary to calculate its effects on TIR transmission. Transmission depends on humidity and air temperature, and for pathlengths through the atmosphere of less than $1 \mathrm{~km}$, it can be assumed that the atmosphere is vertically homogeneous and produces only small errors (Anderson and Wilson, 1984). One way that data on water vapor can be obtained is through standard meteorological soundings via radiosondes, wherein data on atmospheric temperature, barometric pressure, and humidity are transmitted to a ground receiver as the sounding unit progresses upward through the atmosphere.

Atmospheric attenuation involves the absorption and re-emission of radiation in the atmosphere. This results from the atmosphere between the target and the TIR remote sensing platform, and constituent atmospheric components such as water vapor and various gases, having a temperature above absolute zero. As defined by Planck's equation, the atmosphere and its constituents behave as targets themselves, absorbing and emitting radiation within the atmospheric path between the target on the ground and the TIR remote sensing platform. Atmospheric path radiance is independent of the target of interest and adds a finite amount of radiation to that in total reaching the TIR sensor (Anderson, 1992).

Although the effects of atmospheric path transmission and path radiance, if left uncorrected, can provide inaccurate results when deriving surface temperatures from TIR remote sensing data, they can be modeled using computer algorithms. A widely-used atmospheric modeling program is the LOWTRAN soft- 
ware (Kneizys et al., 1983) that has been designed to estimate atmospheric transmission and path radiance for a given atmospheric pathlength over a wavelength region of $0.25-28.5 \mu \mathrm{m}$. The LOWTRAN software permits estimation of atmospheric effects using one of six model atmospheres; these models may be mixed to account for multiple variations for the six standard atmospheric models. Meteorological data may also be input to LOWTRAN to construct a definitive atmospheric profile for a specific site location. Additionally, this atmospheric modeling software contains numerous aerosol models that define the properties of four vertical subdivisions of the Earth's atmosphere. These aerosol models are user-defined to permit a best representation of the atmosphere above the target at the time of TIR data collection (Anderson, 1992). LOWTRAN has produced good results in modeling atmospheric profiles when meteorological data have been used to define atmospheric profile characteristics (Wilson and Anderson, 1986; Holbo and Luvall, 1989; Luvall and Holbo, 1989; Luvall et al., 1990; Anderson, 1992; Quattrochi and Ridd, 1994).

Information from radiosondes input to atmospheric models, such as LOWTRAN, do have drawbacks in that these data may not provide spatial estimates of water vapor; i.e., radiosondes provide point data on atmospheric characteristics that may or may not be representative for atmospheric correction of regional scaled TIR data, for example from the AVHRR satellite. One procedure that has been widely used to correct for atmospheric effects in estimating surface temperature using AVHRR data is the "split window" method (Price, 1984; Dalu, 1986). In the application of the split windows technique, two channels within the atmospheric water vapor window between $10.5-12.5 \mu \mathrm{m}$ from the AVHRR (at $11 \mu \mathrm{m}$ and $12 \mu \mathrm{m}$ which correspond to channels 4 and 5 , respectively) are used to derive temperatures corrected for atmospheric absorption. The brightness temperature differences between the channels $\left(T_{4}-T_{5}\right)$ can then be directly related to the atmospheric absorption due to water vapor. The split window method is described in detail in Price (1984) and Dalu (1986). A concern in the direct application of the split window method, however, is that it works well only for retrieval of sea surface temperatures (SSTs) and is not suitable for direct calculation of temperatures over land. This is because the sea surface can be assumed to be a black body with an emissivity of 1.0 , as opposed to land surfaces which are non-black body radiators with emissivities $<1.0$. In the split window channels of the AVHRR, natural surface emissivities are close to unity, but there is some spectral variation that depends on surface type. Atmospheric and emissivity effects are coupled as a result of downwelling atmospheric radiance at the surface; additionally, the emissivity effect depends on the surface-atmosphere temperature difference. Recent work, for example by Becker and Li (1990), Coll et al., (1994a, 1994b), and Eck and Holben (1994), has addressed the problems associated with using the split window approach for calculating water vapor absorption over land surfaces.

In the analyses of multiple scaled TIR data, it may be seen where atmospheric effects can have a deleterious cascading influence on the prediction and interrelation of temperatures from data acquired by different sensors (i.e., different spatial scales) and at different times. One option is to assume all targets are 
black-bodies, and that the atmosphere does not exist. In this case, it would be possible to calculate relative temperatures for landscape components as computed for TIR data obtained from multiple sensors. Such estimates have some utility for deriving temperature estimates collected over large areas of relatively homogeneous land cover where the variation in emissivity would naturally be low (e.g., agricultural fields, large water bodies) (Anderson, 1985). For most Earth science research purposes, however, this method would provide highly inaccurate temperature estimates for analyses of landscape characteristics and biophysical processes.

A better, but much more resource-intensive method to compensate for the effects of atmosphere, is to obtain atmospheric profiles at the times of TIR data collection. Meteorological data could be used in conjunction with computer algorithms (e.g., LOWTRAN) to model atmospheric effects for data obtained at different times and at different spatial scales, either by the same or with different TIR sensors. The relatively high cost involved in collecting and processing atmospheric profile data, however, is a limitation on obtaining these data in conjunction with multiple scaled TIR data collection. Additionaly, this method is opportunity specific; i.e., it involves considerable pre-data acquisition planning and coordination which also restricts its applicability to intensive joint field and remote sensing data acquisition campaigns, such as that conducted under the auspices of the FIFE program (Sellers et al., 1992).

\section{SPATIAL AND TEMPORAL MODELING WITH MULTIPLE SCALED TIR DATA}

It is apparent that the extraction of accurate surface temperature measurements from multiple scaled TIR data is not straightforward, given the challenges in working with TIR data as described here. Still, it is obvious that the prospect of using multiple scaled TIR data for analyses of thermal responses and energy flux relationships for landscape and surface-atmosphere interactions remains high. Most importantly, it is not the sole use of multiple scaled TIR data in analysis of thermal responses that is the real virtue of applying a multiple scaled approach to a variety of Earth-science problems. It is the employment of multiple scaled TIR data in modeling scenarios of land-surface thermal energy dynamics that offers the most significant application of these data.

As noted by Strahler et al. (1986), a remote sensing model may be generalized as having three components: (1) a scene model, which specifies the form and nature of the energy and matter within the scene and their spatial and temporal order; (2) an atmospheric model, which describes the interaction between the atmosphere and the energy entering and being emitted from the scene; and (3) a sensor model, which describes the behavior of the sensor in responding to the energy fluxes incident upon it and in producing the measurements that constitute the image. Attention has been already given to the importance of atmospheric and radiometric calibration of TIR data within a multiple spatial and temporal scaled context (e.g., atmospheric and sensor models). Thus, it is useful to use the concept of the scene model to discuss the significance of multiple scaled TIR, both 
in space and time, for developing more robust models of landscape and Earthsystem processes.

Questions of modeling multiple scaled TIR data inherently revolve around the aspects of spatial scale, scale invariance, and autocorrelation of the phenomena under investigation. It is useful, therefore, to conceive of the scene models described by Strahler et al. (1986) as $H$-resolution and $L$-resolution models, which reflect the extant relationships between the size of the elements (i.e., the target or targets of interest) in the scene model and the GRC recorded by the sensor. In an $H$-resolution model, the elements of the scene are larger than the GRCs; conversely, an $L$-resolution model has scene elements that are smaller than the GRCs.

Other factors that affect modeling of landscape phenomena and processes or land-atmosphere interactions using multiple scaled TIR data focus on whether the characteristics under observation can be modeled deterministically or empirically. A deterministic remote sensing model employs basic physical laws concerning electromagnetic radiation and, in the case of TIR data, the thermodynamics of matter. The formulation of deterministic models specifies terms that include properties and parameters of elements and describe real processes of energy and matter interaction. An empirical remote sensing model relates observed sensor measurements with scene elements, typically in a statistical fashion (Strahler et al., 1986). A classic example of empirical models in remote sensing are the statistical procedures used in developing multispectral classifications of remote sensing data, such as maximum likelihood or probability density function techniques. For modeling of multiple scaled TIR data, deterministic models are most important because they attempt to associate real thermal radiance properties of the phenomenon or process being studied with those recorded by the TIR remote sensing system. Given this theoretical background of remote sensing models, it is useful to explore, at least from a general perspective, some potential avenues where multiple scaled TIR data can be used in developing improved or new models of landscape and surface atmosphere interactions.

\subsection{Multiple Scaled TIR Models: Prospects and Caveats}

Past research into modeling TIR remote sensing data has focused on the derivation of $H$ - and $L$-resolution models for specific phenomena or processes. Hence, TIR measurements have been made at the characteristics scales most opportunistic to the deterministic analysis of surface thermal energy responses or fluxes. Numerous studies have been conducted using hand-held, aircraft, and satellite TIR sensors for measurement of biophysical responses of vegetation, such as soil moisture, plant moisture stress, crop yields, and evapotranspiration (see, for example, Fuchs and Tanner, 1966; Fuchs et al., 1967; Bartholic et al., 1972; Kimes et al., 1980; Hatfield et al., 1984; Reginato et al., 1985; Choudhury, 1989; Kustas et al., 1989; Fuchs, 1990; Vining and Blad, 1992). These same thermal response issues are of concern to those performing research on more diverse natural landscapes (Balick and Wilson, 1980; Fritschen et al., 1982; Taconet et al., 1986; Running, 1991; Nemani et al., 1993). The prospect of modeling surface temperature 
and energy flux relationships using multiple scaled TIR data, however, offers the potential for developing integrative models that heretofore were not available for a broad spectrum of landscape processes and land-atmosphere interactions (Curran and Foody, 1994). Moving to integrative models will enable us to incorporate TIR data obtained at different spatial scales and times with ground-based radiometer and meteorological data into a common format, for predicting and modeling across space and time continuums. Thus, multiple scaled TIR models will permit extrapolation across $H$ - and $L$-resolution models to provide measurements of surface thermal gradients and thresholds for landscape processes and land-atmosphere interactions regardless of spatial or temporal scale.

Multiple scaled TIR-based remote sensing models, for example, will be of great utility in furthering the analysis of thermal "cross-over points," both in space and time, for different biophysical processes. These cross-over points result as a function of differential heating and cooling of objects and surface materials and their attendant backgrounds. Periods of cross-over occur when a target's rate of heat emission coincides with that of its background, producing the same thermal radiance response incident upon a TIR remote sensor. The confusion of a target's thermal surface response with that of its background leads to inaccurate measurement of temperatures because dissimilar materials exhibit similar radiant temperatures (Estes et al., 1983; Lynn, 1986). There usually are two diurnal times of cross-over-morning and evening-and it is important to avoid these times in the acquisition of TIR remote sensing data. Employing multiple scaled TIR data, however, will potentially better elucidate both the timing and the critically of these cross-over points, thereby providing times of optimal measurement for analysis of Earth-surface and biophysical phenomena to avoid periods of thermal cross-over. Moreover, as alluded to in Section 3.3, a multiple scaled approach using TIR data will provide insight on whether any thresholds of surface temperatures exist at explicit spatial or temporal scales. These temperature thresholds would be important to the analysis of specific landscape biophysical processes or land-atmosphere interactions that have not been identified using mono-spatial and mono-temporal TIR data (e.g., scaling thresholds at which changes in surface temperatures for a landscape can and cannot be accurately measured).

Additionally, multiple scaled TIR data offer possibilities for developing both an improved understanding and better models of surface energy fluxes. Although results from the FIFE program show that estimating energy fluxes (e.g., sensible heat) from TIR remote sensing data is problematical (Hall et al., 1992), there is substantial evidence that surface energy balances can, with additional work on resolving these problems, be measured using at least mono-scale TIR data (Sader, 1986; Carlson, 1986; Taconet et al., 1986; Luvall and Holbo, 1989; Luvall et al., 1990). Given the prospect for further research into resolving some of the problems experienced in estimating energy fluxes, such as those described in Section 3.3, multiple scaled TIR data may provide insight into the modeling of how energy fluxes change with spatial and temporal scale, as well as how they are affected by the observation of heterogeneous landscapes at different spatial scales. The use of multiple scaled TIR data in estimation of surface energy bal- 
ances would be further enhanced by exploring more completely the relationships between measurements derived from ground-based instruments versus TIR remote sensing data, such those investigated by Moran et al. (1989) and by the FIFE investigators (Sellers et al., 1992; Hall et al., 1992; Sellers and Hall, 1992). The combining of ground-based energy balance estimates with multiple scaled TIR data would provide an integrated data base for modeling spatial and temporal dynamics of surface energy fluxes from both a "bottom-up" and "top-down" approach (Caldwell et al., 1993). Such methods are necessary to appropriately model the influence of landscape characteristics of local and regional climate as an integral part of the current emphasis on understanding the processes and effects of regional and global climate change (Pielke and Avissar, 1990).

\subsection{Absolute and Relative Scale Models and Multlple Scaled TIR Data}

It is unclear whether surface thermal changes at the micro-level, for example, at the plant leaf scale, can be adequately related to the canopy, pixel, and scene levels. Further exploitation of multiple scaled TIR will provide needed insight into modeling how the interrelationships between levels of observation and the functionality of biospheric processes change with space and time scales. This would be beneficial to the successful translation of surface thermal response and energy flux models across scales, such as those needed to address global change issues. Thus, the successful integration of ground-based meteorological data with multiple scaled TIR data would provide simulations that bridge the gap between absolute scale and relative scale models. Absolute scale (or space) describes actual distance, direction, shape, and geometry as defined by a Euclidean-based coordinate system (usually a gridded system), as well as the size of the area under observation (e.g., local, regional, global) (Meentemeyer, 1989). Relative space centers on two considerations: (1) that space is defined by the spatial elements and processes under consideration and (2) that the end result of defining elements or processes may result in the perception of non-Euclidean (i.e., non-gridded) terms (Meentemeyer, 1989). Relative space, therefore, implies a transformation of absolute space to a space that describes the relative distance, direction, or geometry predicated on some functional relationship (e.g., a process-oriented relationship).

Remote sensing observes phenomena from an absolute space perspective, whereby it is relatively easy through image processing techniques to relate the position and size of various phenomena (e.g., land covers) at various spatial scales. Surface temperature and energy flux relationships on the other hand, are process-driven; that is, they result from the direct and indirect thermodynamic factors that control the uptake and dispersion of solar radiation and moisture for specific phenomena or biospheric processes. Hence, the key in understanding and modeling energy flux relationships is to link observations at both absolute and relative scales. Figure 4 provides an illustration of how by using multiple scaled TIR data in conjunction with ground-based meteorological data, it should be theoretically possible to link models of absolute and relative scale processes (see also Quattrochi, 1993). For example, if it is assumed that vertical fluxes 


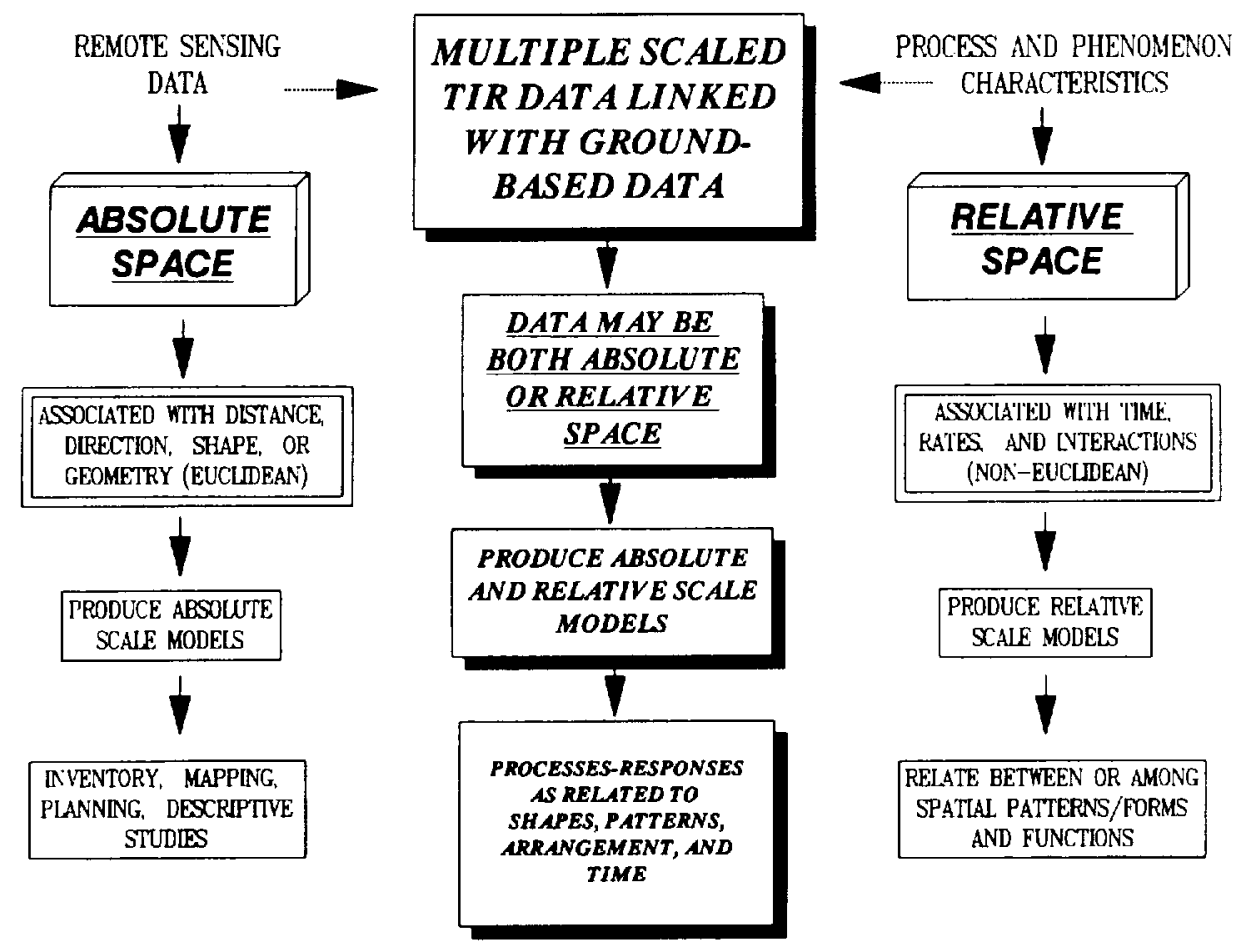

FIGURE 4 An example of the interrelationships of absolute and relative space or scale as they relate to multiple scaled TIR data linked with ground-based data for modeling processes-responses of natural phenomena.

dominate, the energy and moisture balance between the ground and the overlying atmosphere for landscapes are fundamentally functions of vegetation and soil moisture. Although energy budget equations can be written to describe the flux of heat and water into and out of the ground, there are several parameters influencing these budgets that are directly dependent upon soil type and vegetation characteristics. Soil parameters include density, porosity, texture, thermal diffusivity, hydraulic conductivity, and photometric properties. Vegetation parameters include leaf area index as a function of height, stomatal resistance, albedo, aerodynamic roughness, displacement height, percentage coverage, and photometric properties (Pielke and Avissar, 1990). Because the surface heat and water budgets are functions of the values of these parameters, landscape changes in general, will alter the energy balances of the soil and vegetation parameters. The sensitivity of atmospheric conditions to even modest changes in surface characteristics can be illustrated via the Earth's global energy budget

$$
\sigma T_{E}^{4}=S(1-A), \quad\left(\sigma=5.67 \times 10^{-8} \mathrm{Wm}^{-2} \mathrm{~K}^{-4}\right)
$$

where we assume the Earth radiates as a black-body with a temperature $T_{E}, S$ is the solar constant $\left(S=1380 \mathrm{Wm}^{-2}\right)$, and $A$ is albedo of the surface. Thus, 
equation (12) illustrates the sensitivity of the Earth's climate to even very small changes in land use (Pielke and Avissar, 1990). To develop complete models of this sensitivity, however, requires that we link absolute scale observations via remote sensing (i.e., measurement at different scales using TIR data) with relative scale processes (e.g., changes in vegetation and soil parameters in response to thermodynamic input). Although the cause and effect processes inherent to landscape change and energy flux dynamics may appear close in absolute space (e.g., the identification of a specific landscape or landscape component as a source of change in soil moisture), these factors may be very distant in relative space (i.e., the dynamics of the attendant changes in energy flux processes in response to changes in soil moisture) when time, rates, and interactions are considered (e.g., the rate of change in soil moisture and evapotranspiration as a function of the distribution and arrangement of land covers across a landscape). The determination of the scale at which a process or phenomenon operates, therefore, is difficult to identify in terms of relative space and it would be better to transform data residing in absolute space (i.e., remote sensing data) to units based on a relative space to understand the spatial thresholds inherent to process-response mechanisms as they are manifested at multiple scales. Thus, by combining multiple scaled TIR data with other data as illustrated in Figure 4, it would be possible to understand the synergistic linkages between components of the landscape with energy flux dynamics to provide a more complete quantitative of landatmosphere interactions as they are reflected at different spatial and temporal scales.

\section{CONCLUDING REMARKS}

It is obvious that the path to the development of robust integrative models using multiple scaled TIR data is a challenging one. Perhaps the greatest difficulties in modeling multiple scaled TIR data arise from the scale-dependent nature of landscape characteristics. Because landscape heterogeneity increases at finer spatial scales, the concomitant surface thermal responses and energy fluxes also become more complex. Thus, modeling of the sensitivity of fluxes to changes in land-surface characteristics requires knowledge of natural spatial autocorrelations and the patterns and distributions of landscape elements; i.e., are they disperse, aggregated, or random? As we have seen, the arrangement of the landscape has a paramount influence on the observation, detection, and measurement of surface thermal responses and energy flux dynamics. The employment of multiple space and time scaled TIR data in modeling thermodynamic attributes and energetics, therefore, appears as a salient method for developing a better understanding of the process-response mechanisms associated with different landscapes and the changes of these landscapes over time.

Although the prospects of using multiple scaled TIR data to address questions of landscape surface energy dynamics and land-atmosphere interactions are enticing, there are a number of technical and theoretical difficulties that must be more thoroughly investigated to bring the potential benefits of multiple scaled 
TIR data to fruition. Notwithstanding the difficulties associated with determining emissivities, the incorporation of TIR data obtained from the same and different sensing systems, and at differing spatial and temporal scales, must contend with the problems associated with scene versus sensor spatial frequencies and non-linearities in surface temperatures. In dealing with the manipulation of multiple scaled TIR data sets we must delve further into the questions associated with how the atmosphere affects these data. It must also be noted that there is a substantial increase in costs associated with using multiple scaled TIR data. These costs accrue to the increase in money required to collect or purchase TIR data obtained at different spatial or temporal scales (either from the same or different sensors), in the time needed to manipulate and analyze these data, and in the computing resources needed to process multiple scaled data. These costs may be considerable, depending upon the extent of the data needed to support overall research objectives (e.g., field and remote sensing data collection and analyses to support a major TIR research campaign such as that illustrated in the FIFE program are very expensive). Moreover, it is the analytical problems that are most perplexing-those related to neighborhood constraints and scale invariance-which produce perturbations in the analysis of TIR data obtained at different space and time scales, and these pose important challenges to the overall applicability of a multiple scaled approach using thermal remote sensing data.

In contemplation of these factors, however, we must not lose sight of the fact that landscape surface-atmosphere thermal interactions are integral to the operation of the Earth's energy budget. A key component in understanding how the Earth's climate is changing, as influenced by anthropogenic and natural sources, is to quantitatively evaluate the thermal energy processes that drive these changes and the scale dependencies of these changes. Investigations into the processesresponses associated with global change will be greatly impaired without understanding how to effectively utilize multiple scaled thermal IR data available from current sensing systems, as well as for the planning and development of more advanced TIR platforms. Ultimately, this may provide critical information for understanding how the global climate system functions as an integrated system.

\section{References}

Anderson, J. E. (1985) Thermal infrared data: Its characteristics and use. In Technical Papers, First Annual Joint Symposium, Gulf Coast Chapters of the Hydrographic Society and the American Society for Photogrammetry and Remote Sensing, Long Beach, MS, 143-155.

Anderson, J. E. (1992) Determination of water surface temperature based on the use of thermal infrared multispectral scanner data. GeoCarto International 7 (3): 3-8.

Anderson, J. M. and Wilson, S. B. (1984) The physical basis of current infrared remote-sensing techniques and the interpretation of data from aerial surveys. International Journal of Remote Sensing 5 (1): $1-18$.

Artis, D. A. and Carnahan, W. H. (1982) Survey of emissivity variability in thermography of urban areas. Remote Sensing of Environment 12: 313-329.

Balick, L. K. and Wilson, S. K. (1980) Appearance of irregular tree canopies in nighttime highresolution thermal infrared imagery. Remote Sensing of Environment 10: 299-305.

Baraniak, D. W. (1983) Thermal remote sensing. In Introduction to Remote Sensing of the Environment, 2nd cd. (B. F. Richason, Jr., ed.). Dubuque, IA: Kendall/Hunt Publishing Co., 167-190. 
Bartholic, J. F., Namken, L. N., and Wiegand, C. L. (1972) Aerial thermal scanner to determine temperatures of soils and of crop canopies differing in water stress. Agonomy Journal 64: 603608.

Becker, F. and $\mathrm{Li}, \mathrm{Z}$-L. (1994) Towards a local split window method over land surfaces. International Journal of Remote Sensing 11 (3): 369-393.

Burnay, S. G., Williams, T. L. and Jones, C. H. (eds.) (1988) Applications of Thermal lmaging. Bristol, England: Adam Hilgar.

Caldwell, M. M., Matson, P. A., Wessman, C. and Gamon, J. (1993) Prospects for scaling. In Scaling of Physiological Processes: Leaf to Globe (J. R. Ehleringer and C. B. Field, eds.). San Diego: Academic Press, 223-230.

Carlson, T. N. (1986) Regional-scale estimates of surface moisture availability and thermal inertia using remote thermal measurements. Remote Sensing Reviews 1: 197-247.

Choudhury, B. J. (1989) Estimating evaporation and carbon assimilation using infrared temperature data: Vistas in modeling. In Theory and Applications of Optical Remote Sensing (G. Asrar, ed.). New York: John Wiley and Sons, 628-690.

Coll, C., Caselles, V., Sobrino, J. A. and Valor, E. (1994a) On the atmospheric dependence of the split-window equation for land surface temperature. International Journal of Remote Sensing 15 (1): 105-122.

Coll, C., Caselles, V. and Schmugge, T. J. (1994b) Estimation of land surface emissivity differences in the split window channels of the AVHRR. Remote Sensing of Environment 48: 127-134.

Colwell, R. N. (ed.) (1983) Manual of Remote Sensing (2nd ed.), Vol. 1, Theory, Instruments, and Techniques. Falls Church, VA: ASPRS.

Curran, P. J. and Foody, G. M. (1994) Environmental issues at regional to globe scales. In Environmental Remote Sensing from Regional to Global Scales (G. M. Foody and P. J. Curran, eds.). New York: John Wiley and Sons, 1-7.

Dalu, G. (1986) Satellite remote sensing of atmospheric water vapour. International Journal of Remote Sensing 7 (9): 1089-1097.

Davis, F. W. and Simonett, D. S. (1991) GIS and remote sensing. In Geographical Information Systems: Principles and Applications. Vol. I: Principles. New York: Longman Scientific and Technical, 191213.

Davis, F. W., Quattrochi, D. A., Ridd, M. K., Lam, S.-N., Walsh, S. J., Michaelsen, J. C., Franklin, J., Stow, D. A., Johannsen, C. J. and Johnston, C. A. (1991) Environmental analysis using integrated GIS and remotely sensed data: Some research needs and priorities. Photogrammetric Engineering and Remote Sensing 57 (6): 689-697.

Duggin, M. J. and Robinov, C. J. (1990) Assumptions implicit in remote sensing data acquisition and analysis. International Journal of Remote Sensing 11 (10): 1669-1694.

Ehleringer, J. R. and Field, C. B. (1993) Scaling Physiological Processes: Leaf to Globe. San Diego: Academic Press.

Eck, T. F. and Holben, B. N. (1994) AVHRR split window temperature differences and total precipitable water over land surfaces. International Journal of Remote Sensing 15 (3): 567-582.

Estes, J. E., Hajic, E. J. and Tinney, L. R. (1983) Fundamentals of image analysis: Analysis of visible and thermal infrared data. In Manual of Remote Sensing, 2nd ed. (R. N. Colwell, ed.). Falls Church, VA: American Society for Photogrammetry and Remotc Sensing, 987-1124.

Foody, G. M. and Curran, P. J. (1994) Scale and environmental remotc sensing. In Environmental Remote Sensing from Regional to Global Scales (G. M. Foody and P. J. Curran, eds.). New York: John Wiley and Sons, 223-232.

Fritschen, L. J., Balick, L. K. and Smith, J. A. (1982) Interpretation of infrared nighttime imagery of a forested canopy. Journal of Applied Meteorology 21: 730-734.

Fuchs, M. and Tanner, C. B. (1966) Infrared thermometry of vegetation. Agronomy Journal 58: 597601.

Fuchs, M., Kanemasu, E. T., Kerr, J. P. and Tanner, C. B. (1967) Effect of viewing angle on canopy temperature measurements with infrared thermometers. Agronomy Journal 59: 494-496.

Fuchs, M. (1990) Canopy thermal infrared observations. Remote Sensing Reviews 5 (1): 323-333.

Garratt, J. R., Krummel, P. B. and Kowalczyk, E. A. (1993) The surface energy balance at local and regional scales-A comparison of General Circulation Model Results with Obscrvations. Journal of Climate 6 (6): 1090-1109.

Gerstl, S. A. W. and Simmer, C. (1986) Radiation physics and modeling for off-nadir satellite-sensing of non-lambertian surfaces. Remote Sensing of Environment 20: 1-29.

Goetz, S. J., Hall, F. G., Markham, B. L. and Dubayah, R. O. (1993) Inter-comparison of retrieved surface temperature from multi-resolution sensors at the FIFE site. In Technical Papers, Vol. II, ASPRS/ACSM Annual Meeting, New Orleans, LA: 108-117. 
Goetz, S. J., Halthorne, R., Hall, F. G. and Markham, B. L. (1994) Surface temperature retricval from multi-resolution sensors at the FIFE site. Journal of Geophysical Research. FIFE Special Issue II (in press).

Hall, F. G., Strebel, D. E. and Sellers, P. J. (1988) Linking knowledge among spatial and temporal scale: Vegetation, atmosphere, climate and remote sensing. Landscape Ecology 2 (1): 3-22.

Hall, F. G., Hummerich, K. F, Goetz, S. J., Sellers, P. J. and Nickeson, J. E. (1992) Satellite remote sensing of surface energy balance: Success, failures, and unresolved issues in FIFE. Journal of Geophysical Research 97 (D17): 19,061-19,089.

Harvey, D. (1969) Explanation in Geography. New York: St. Martin's Press.

Hatfield, J. L., Reginato, R. J. and Idso, S. B. (1984) Evaluation of canopy temperature-evapotranspiration models over various surfaces. Agricultural and Forest Meteorology 32: 41-53.

Holbo, H. R. and Luvall, J. C. (1989) Modeling surface temperature distributions in forest landscapes. Remote Sensing of Environment 27: 11-24.

Hook, S. J. and Kahle, A. B. (1990) Techniques for the extraction of emissivity variations from multispectral thermal infrared data. In Proceedings, 10th Annual IGARSS Symposium, May 20-24, College Park, MD, 983-989.

Hummer-Miller, S. (1990) Techniques for noise removal and registration of TIMS data. Photogrammetric Engineering and Remote Sensing 56 (1): 49-53.

Kahle, A. B., Schieldge, J. P. and Alley, R. E. (1984) Sensitivity of thermal inertia calculations to variations in environmental factors. Remote Sensing of Environment 16 (3): 211-232.

Kahle, A. B. and Alley, R. E. (1985) Calculation of thermal inertia from day-night measurements separated by days or weeks. Photogrammetric Engineering and Remote Sensing 51 (1): 73-75.

Kahle, A. B. (1987) Surface emittance, temperature, and thermal inertia derived from Thermal Infrared Multispectral Scanner (TIMS) data for Death Valley, CA. Geophysics 52 (7): 858-874.

Kaufman, Y. J. (1982) Solution of the equation of radiative transfer for remote sensing over nonuniform surface reflectivity. Journal of Geophysical Research 87: 4137-4147.

Kaufman, Y. J. (1984) Atmospheric effect on spatial resolution of surface imagery. Applied Optics 23: $3400-3408$.

Kaufman, Y. J. (1989) The atmospheric effect on remote sensing and its correction. In Theory and Applications of Optical Remote Sensing (G. Asrar, ed.). New York: John Wiley and Sons, 336-428.

Kimes, D. S., Idso, S. B., Pinter, P. J., Jr., Reginato, R. J. and Jackson, R. D. (1980) View angle effects in the radiometric measurement of plant canopy temperatures. Remote Sensing of Environment 10: $273-284$.

Kncizys, F. X., Shettle, E. P., Gallery, W. O., Chetwynd, J. J., Jr., Abreu, L. W., Selby, J. E. A., Clough, S. A. and Fenn, R. W. (1983) Atmospheric Transmittance/Radiance: Computer Code LOWTRAN 6. U.S. Air Force Geophysics Laboratory, Environmental Research Papers No. 846 (AFGL-TR83-0187), Hanscom Air Force Base, MA.

Kustas, W. P., Jackson, R. D., and Asrar, G. (1989) Estimating surface energy-balance components from rcmotely sensed data. In Theory and Applications of Optical Remote Sensing (G. Asrar, ed.). New York: John Wiley and Sons, 604-627.

Lee, A. C. L. (1973) A study of the continuum absorption within the 8-13 $\mu \mathrm{m}$ window. Quarterly Journal of the Royal Meteorological Society 99: 490.

Luvall, J. C. and Holbo, H. R. (1989) Measurements of short-term thermal responses of coniferous forest canopies using thermal scanner data. Remote Sensing of Environment 27: 1-10.

Luvall, J. C., Lieberman, D., Lieberman, H., Hartshorn, G. S. and Peralta, R. (1990) Estimation of tropical forest canopy temperatures, thermal response numbers, and evapotranspiration using an aircraft-based thermal sensor. Photogrammetric Engineering and Remote Sensing 56 (10): 13931401.

Lynn, D. W. (1986) Monotemporal, multitemporal, and multidate thermal infared data acquisition from satellites for soil and surface-material survey. International Journal of Remote Sensing 7 (2): 213-231.

Markham, B. L. and Barker, J. L. (1986) Landsat MSS and TM post-calibration dynamic ranges, cxoatmospheric reflectances and at-satellite temperatures. EOSAT Landsat Technical Note 1: 3-8.

Meentemeyer, V. (1989) Geographical perspectives of space, time, and scale. Landscape Ecology 3 (3/4): $163-173$.

Moik, J. G. (1980) Digital Image Processing of Remotely Sensed Images. NASA SP-431, Washington, DC: NASA Scientific and Technical Information Branch.

Moran, M. S., Jackson, R. D., Raymond, L. H., Gay, L. W. and Slater, P. N. (1989) Mapping surface cnergy balance components by combining Landsat Thematic Mapper and ground-based metcorological data. Remote Sensing of Environment 30: 77-87. 
Nemani, R., Pierce, L. and Running, S. (1993) Developing satellite-derived estimates of surface moisture status. Joumal of Applied Meteorology 32: 548-557.

Pielke, R. A. and Avissar, R. (1990) Influence of landscape structure on local and regional climate. Landscape Ecology 4 (2/3): 133-155.

Price, J. C. (1985) On the analysis of thermal infrared imagery: The limited utility of apparent thermal inertia. Remote Sensing of Environment 18: 59-73.

Price, J. C. (1984) Land surface temperature measurements from the split window channels of the NOAA-7 Advanced Very High Resolution Radiometer. Journal of Geophysical Research 89 (D5): $7231-7237$.

Price, J. C. (1989) Quantitative aspects of remote sensing in the thermal infrared. In Theory and Applications of Optical Remote Sensing (G. Asrar, ed.). New York: John Wiley and Sons, 578603.

Quattrochi, D. A. (1993) The need for a lexicon of scale terms in integrating remote sensing data with geographic information systems. Journal of Geography 92 (5): 206-212.

Quattrochi, D. A. and Ridd, M. K. (1994) Measurement and analysis of thermal energy responses from discrete urban surfaces using remote sensing data. International Journal of Remote Sensing 15 (10): 1991-2022.

Reginato, R. J., Jackson, R. D. and Pinter, J. R., Jr. (1985) Evapotranspiration calculated from remote multispectral and ground station meteorological data. Remote Sensing of Environment 18: 75-89.

Running, S. W. (1991) Computer simulation of regional evapotranspiration by integrating landscape biophysical attributes with satellite data. In Land Surface Evaporation: Measurement and Parameterization (T. J. Schmugge and J.-C. André, eds.). New York: Springer-Verlag, 359-369.

Sader, S. A. (1986) Analysis of effective radiant temperatures in a Pacific Northwest forest using Thermal Infrared Mult ispectral Scanner data. Remote Sensing of Environment 19: 105-115.

Schott, J. R. (1989) Image processing of thermal infrared images. Photogrammetric Engineering and Remote Sensing 55 (9): 1311-1321.

Sellers, P. J. and Hall, F. G. (1992) FIFE in 1992: Results, scientific gains, and future research directions. Journal of Geophysical Research 97 (D17): 19,091-19,109.

Sellers, P. J., Hall, F. G., Asrar, G., Strebel, D. E. and Murphy, R. E. (1992) An overview of the First International Satellite Land Surface Climatology Project (ISLSCP) Field Experiment (FIFE) Journal of Geophysical Research 97 (D17): 18,345-18,371.

Short, N. M. and Stuart, L. M., Jr. (1982) The Heat Capacity Mapping Mission (HCMM) Anthology. NASA SP-465. Washington, DC: NASA Scientific and Technical Information Branch.

Strahler, A. H., Woodcock, C. E. and Smith, J. A. (1986) On the nature of models in remote sensing. Remote Sensing of Environment 20: 121-139.

Taconet, O., Carlson, T., Bernard, R. and Vidal-Madjar, D. (1986) Evaluation of a surface/vegetation parameterization using satellite measurements of surface temperature. Journal of Climate and Applied Meteorology 25 (11): 1752-1767.

Turner, M. G., O'Neill, R. V. and Milne, B. T. (1989a) Effects of changing spatial scale on the analysis of landscape patterns. Landscape Ecology 3 (3/4): 153-162.

Turner, M. G., Dale, V. H. and Gardner, R. H. (1989b) Predicting across scales: Theory, development and testing. Landscape Ecology 3 (3/4): 245-252.

Vining, R. C. and Blad, B. L. (1992) Estimation of sensible heat flux from remotely sensed canopy temperatures. Journal of Geophysical Research 97 (D17): 18,951-18,954.

Wilson, S. B. and Anderson, J. M. (1986) The applicability of LOWTRAN 5 computer code to aerial thermographic data collection. International Journal of Remote Sensing 7 (3): 379-388. 